\title{
Advanced Design and Manufacture of Mechanoactive Materials Inspired by Skin, Bones, and Skin-on-Bones
}

\author{
Melissa Louise Knothe Tate* \\ Inaugural Paul Trainor Chair of Biomedical Engineering, Director MechBio Team, Graduate School of Biomedical \\ Engineering, Faculty of Engineering, University of New South Wales, Sydney, NSW, Australia
}

Life is mechanobiological. Natural living materials exhibit remarkable, emergent and smart properties under mechanical loading. Such materials are classified as mechanoactive, in contrast to electroactive polymers and materials that exhibit advanced properties when subjected to electrical stimulation. Cutting edge, multiscale imaging technologies have proven enabling for the elucidation of molecular to mesoscale structure and function of natural mechanoactive materials. Using MicroscopyAided Design And ManufacturE, (MADAME) this perspective article describes mechanoactive properties of natural materials including skin-on-bones (periosteum)

OPEN ACCESS

Edited by:

Chi Zhou,

University at Buffalo, United States

Reviewed by:

PaYaM ZarrinTaj,

Oklahoma State University,

United States

Zeyu Chen,

Central South University, China

Xianfeng Lin,

Zhejiang University, China

${ }^{*}$ Correspondence: Melissa Louise Knothe Tate m.knothetate@unsw.edu.au

Specialty section: This article was submitted to Biomaterials,

a section of the journa Frontiers in Bioengineering and Biotechnology

Received: 06 March 2020

Accepted: 30 June 2020

Published: 25 August 2020

Citation:

Knothe Tate ML (2020) Advanced

Design and Manufacture of Mechanoactive Materials Inspired by Skin, Bones, and Skin-on-Bones.

Front. Bioeng. Biotechnol. 8:845.

doi: 10.3389/fbioe.2020.00845 and bone itself. In so doing, it demonstrates the principle to emulate natural smart properties using recursive logic, the basis of many computer algorithms, and to design and manufacture mechanoactive materials and products using advanced manufacturing methods that also incorporate principles of recursive logic. In sum, the MADAME approach translates physically the computer science paradigm of recursion by implementing Jacquard textile methods, which themselves form a historical basis for computing machines, together with additive manufacturing methods including multidimensional printing, stereolithography, laser sintering, etc. These integrated methods provide a foundation and translational pathway for scaled-up manufacture of disruptive mechanoactive materials that will find use in fields as varied as medicine, safety, transport and sports, for internal (implants) and external (wearables) applications.

Keywords: Microscopy-Aided Design And ManufacturE, mechanoactive materials, composite, biomimicry, additive manufacturing, smart materials, emergent properties, similitude

\section{INTRODUCTION}

Nature abounds with stimuli-responsive, so-called smart materials. Examples of such materials, at the macro- to meso-length scale, include skin, bones, and skin-on-bones (periosteum) in the animal kingdom, and eucalyptus tree bark, cambium, and wood in the plant kingdom. Connective tissues comprising skin and other soft animal tissues exhibit remarkable mechanical strength, functional barrier properties to prevent moisture loss to the environment, while also "waterproofing" the internal organs, as well as self-healing and -sensing (e.g., pressure sensing) capacities (Knothe Tate et al., 2009; Tee et al., 2012). Vascular tissues of trees generate hydraulic pressure pulses when they bend in the wind (Lopez et al., 2014) and bone exhibits flow directing properties

Abbreviations: EMT, Epithelial to Mesenchymal Transition; MADAME, Microscopy-Aided Design And ManufacturE; MET, Mesenchymal to Epithelial Transition; Multi-D, Multi-Dimensional. 
under mechanical loading, emerging from different calibers of interconnected vascular, pericellular, and matrix porosities (Knothe Tate et al., 2009). While top-down approaches to designing and manufacturing such smart materials have met with little success, bottom-up approaches using paradigms of "cellular manufacture" have been met with great success (Knothe Tate, 2011a,b; Tee et al., 2012; Knothe Tate, 2017b; Sidler et al., 2018).

Remarkably, the "brainless" cells that manufacture all of the aforementioned smart materials, themselves form living sensors, actuators and transducers at the nano- to micron length scale (Knothe Tate and Niederer, 1998; Mishra and Knothe Tate, 2003; Knothe Tate and Anderson, 2013; Knothe Tate et al., 2016b; Ng et al., 2019, 2020). The advent of imaging across length- and time-scales has enabled not only unprecedented elucidation of the mechanisms underpinning cells' and natural materials' smart properties (Figure 1), but also the design and manufacture of new materials emulating nature's own (Figure 2; Knothe Tate, 2017b). MADAME refers to a computer-aided additive manufacturing platform that incorporates Multi-D printing and/or computercontrolled weaving to create novel, bio-inspired materials and products (Steck et al., 2000; Knothe Tate, 2003; Sidler et al., 2018; $\mathrm{Ng}$ et al., 2019). The state of the art imaging capacity enables observation of live cells in their native tissue habitats. Design thinking processes empower engineers to empathize with their cells, imagining and feeling what they experience and envisioning their responses. In empathizing with their cells, engineers may be better equipped to prototype mechanoactive materials and architectures as cells do, from raw materials that they themselves produce and adapting their own structure and function, and ultimately their own niche to survive (Knothe Tate et al., 2016b).

This perspective article expands upon and integrates these topics to establish a foundation for advanced design and manufacture of mechanoactive materials that will be relevant for fields of use as varied as the medical and transport sectors, as well as for external and internal applications.

\section{CELLS AS SENSORS, ACTUATORS, AND TRANSDUCERS}

Throughout nature, cells are the master designers, manufacturers and builders of tissue architectures underpinning e.g., trees and their population forests in the plant kingdom, as well as organs and organismal systems in the animal world (Knothe Tate et al., 2010). Cells literally differentiate themselves in their degree of differentiation, a biological term for structural and functional specialization. From undifferentiated stem cells, to terminally differentiated cells as diverse as dendritic bone and brain cells ${ }^{+}$, the structure and architecture of the cell encodes structural and functional memories of the cell's life experience. Repeated activities reinforce structural connections (e.g., of the cytoskeleton, cell-matrix and cell-cell connections, etc.) which are stabilized over time, resulting in an adaptive functional

${ }^{+}$In contrast to undifferentiated cells, highly specialized, terminally differentiated cells exert low potential to become different specialized cell types, a process that can theoretically, albeit rarely, occur through dedifferentiation and redifferentiation, or cross-differentiation. memory of cell's experiences throughout life (Knothe Tate and Fath, 2016; Knothe Tate et al., 2016b; Knothe Tate, 2017a). Cells' dynamic stability and agility are a function of the cells' structural stability as well as adaptive capacity, in context of cellular time scales for e.g., division, motility, structural protein expression and secretion, etc. The cytoskeleton is one repository of cellular information and the totality of cell structures at any given moment in time serve as an integrated sensor for the health state of the tissue and/or organ at that point in time and geographic location (Knothe Tate and Fath, 2016; Knothe Tate et al., 2016b).

Since cells sense their local environment and transduce biophysical (mechanical, electrical, osmotic, etc.) information to the nucleus, where gene up- and down regulation leads to stabilization of the cell and/or the cell's environment over time, the cell itself is also an actuator of structural and architectural change as well as new local and global equilibria (Knothe Tate et al., 2016b; Figure 1C). By sensing and transducing information from its environment to the nucleus, where the basic building block proteins of tissues are created and secreted vectorially (with a magnitude/concentration and direction) (Knothe Tate et al., 2011a; Moore et al., 2014, 2016) to the extracellular matrix, the cell actively influences force balances at all relevant interfaces - between cells, cells and the matrix, and even between the cytoplasm and the nucleus. Cells are indeed smart in a materials science context, if not in a brain science context.

\section{TISSUES CELLS WEAVE AND THEIR SMART PROPERTIES}

Natural materials such as animal and plant tissues are natural composites comprising resilient collagens (animals) and celluloses (plants) that confer toughness, elastin (animals) and elastin-like polypeptides (plants) that impart elasticity, and proteoglycans that bind water and give turgidity. Cells "spin and weave" components of tissues in situ (Knothe Tate, 2011b, 2017b; $\mathrm{Ng}$ et al., 2017c) - the remarkable stimuli-responsive (smart) and adaptive properties of tissues emerge macroscopically from the directional, cellular secretion of nanoscopic extracellular matrix proteins as well as their anisotropic multicellular assembly and dis-assembly (polymerization and depolymerization). While in the future, the capacity to guide cells to manufacture smart materials in a controlled way may become possible, the current focus of this author's R\&D program is on emulating nature's paradigms, either through scaled-up solutions based on similitude theory (Anderson et al., 2008; Knothe Tate, 2017b) or recursive logic based approaches (Steck et al., 2000; Knothe Tate, 2017b; Ng et al., 2017c). An overview is provided below, with specific examples inspired by skin, bone, and skin-on-bones in the following sections.

\section{Geometry}

The success of Lego building blocks, with their universal interlocking mechanism, is their flexibility to build structures only limited by the creative capacity of the human brain. Nature's counterpart could be the cells' capacity to organize into sheets (epithelial sheet, Figure 1A) and three-dimensional, 

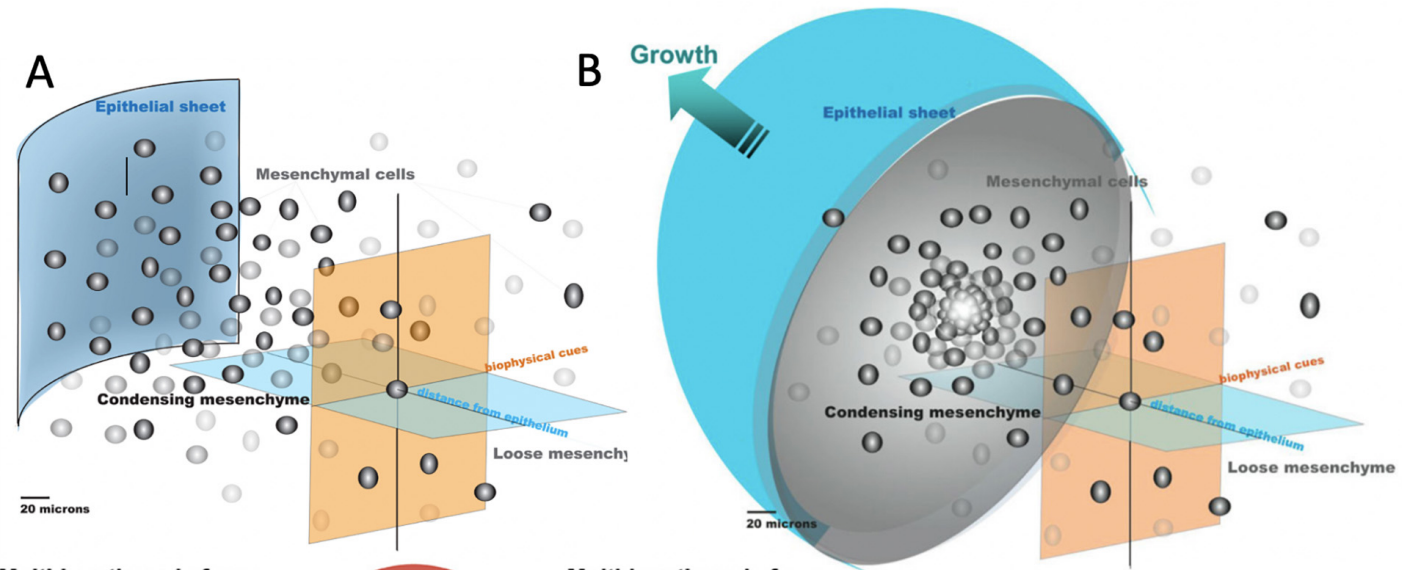

\section{Multi-length scale force internalization and mechanotransduction

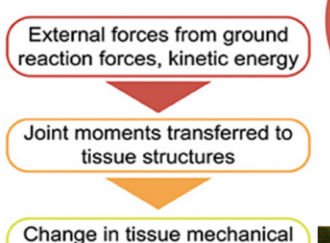

Change in tissue mechanical state due to loading behavior
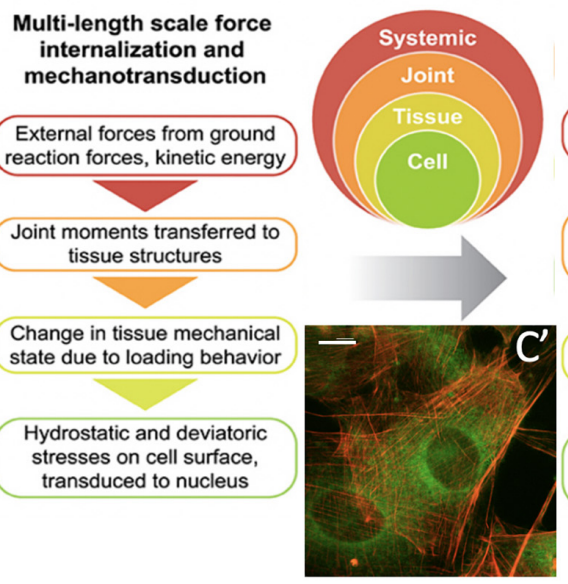

Multi-length scale force
mechanobiology driven repair and rehabilitation

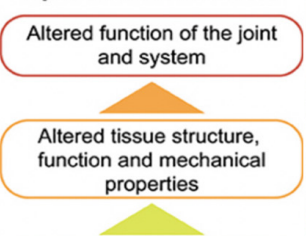

Altered extracellular matrix surrounding cells

\section{Altered protein synthesis/ transcription changing cell behavior/fate}
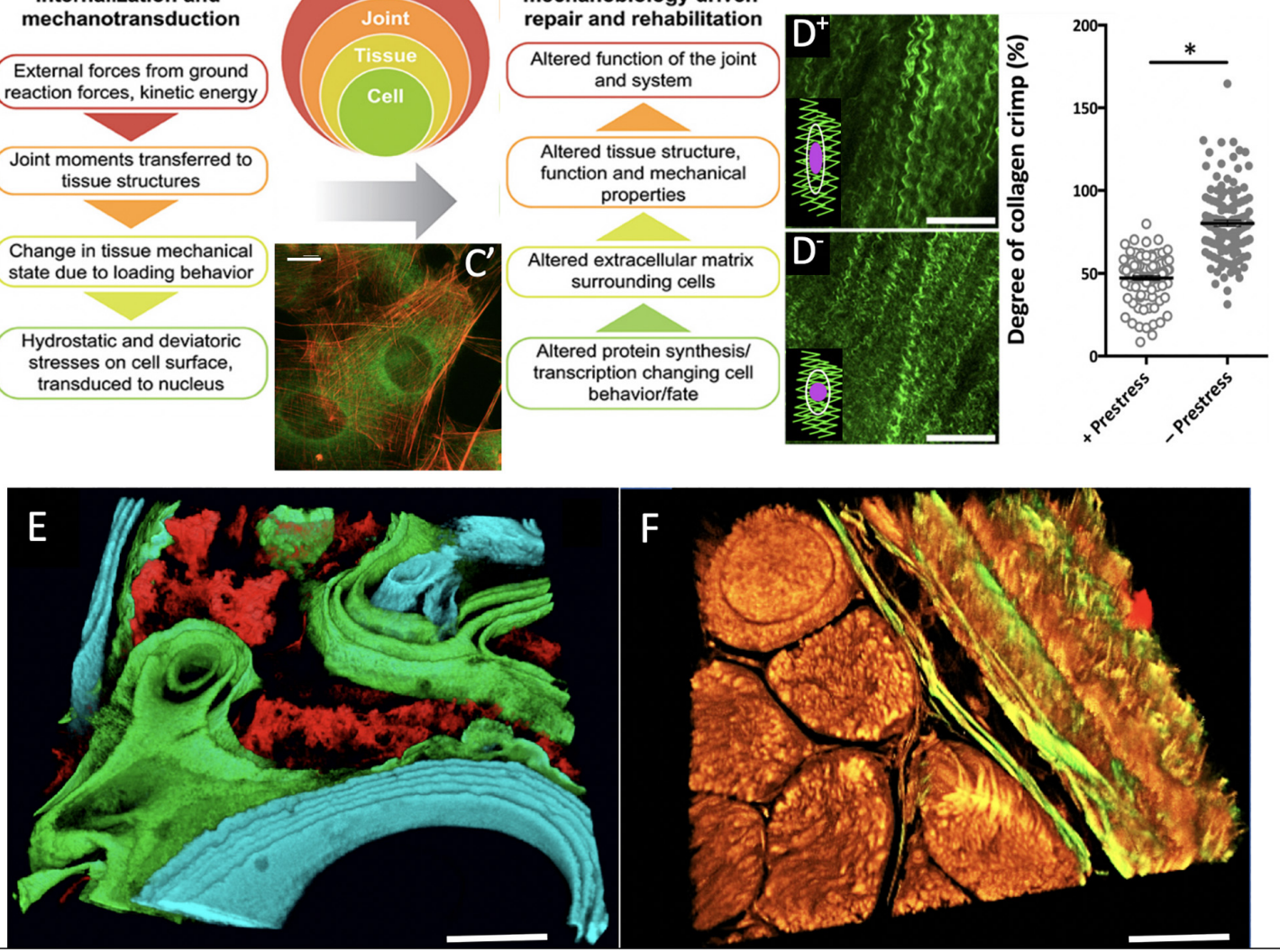

FIGURE 1 | Imaging based studies to elucidate multi-time and length scale properties of tissues, using bone and skin-on-bones (periosteum) as examples. Transitions between Epithelial Sheets (A) and Mesenchyme (B) occur throughout life, providing tissue geometries for functional boundaries and tissue structures (Knothe Tate et al., 2008). (C) At every length scale, mechanical forces are transduced to and sensed by cells, which serve as actuators to build structural proteins that confer toughness, elasticity and turgor to tissue constructs ( $\mathrm{Ng}$ et al., 2017b). (C') After exposure to subtle cues such as 0.8 Pa fluid flow, cells buttress themselves by adapting their cytoskeleton (actin in red) and manufacturing structural proteins that are secreted to the extracellular matrix (collagen autofluorescing in green), thus modulating the cell's own local environment (McBride et al., 2008). Scalebar: $5 \mu m$. ( $\mathbf{D}^{+}$) Once collagens are fully assembled in the extracellular matrix, they resemble curled or crimped structures which provide a scaffold for adherence of quiescent stem cells. Here, in the matrix of the periosteum, the tissue is tacked

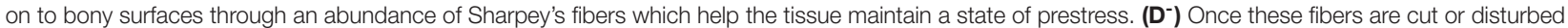
through periosteal lifting surgery or trauma, the prestress in the tissue relaxes; (see graph at right) and the collagen crimp increases significantly when prestress is removed, resulting in rounding of cells, a hypothetical trigger for stem cell egression to sites of injury (Yu et al., 2017). In this way, the "brainless" cells and the matrix interact as smart sensors and transducers and actuators. (E) When administered at known points in time, mineral chelating fluorochromes mark areas of mineralization which can be decoded to show temporal dynamics of mineralization under specific excitation and emission frequencies of light (Knothe Tate, $2011 \mathrm{~b})$. Scalebar: $50 \mu \mathrm{m}$. (F) Mineral nucleates around collagens. The collagens (yellow) and elastins (green) making up the tissue demonstrate the textile structure of tissues (muscle fascicles in lower left quadrant with bone weave in upper right quadrant, and periosteum in between) (Ng et al., 2017c). Together with the organic and inorganic extracellular matrix components, the tissue represents a complex composite structure (Knothe Tate, 2016, 2018, 2019). Scalebar: 50 $\mu$ m. Figure adapted and compiled from previously published figures, with permission. 


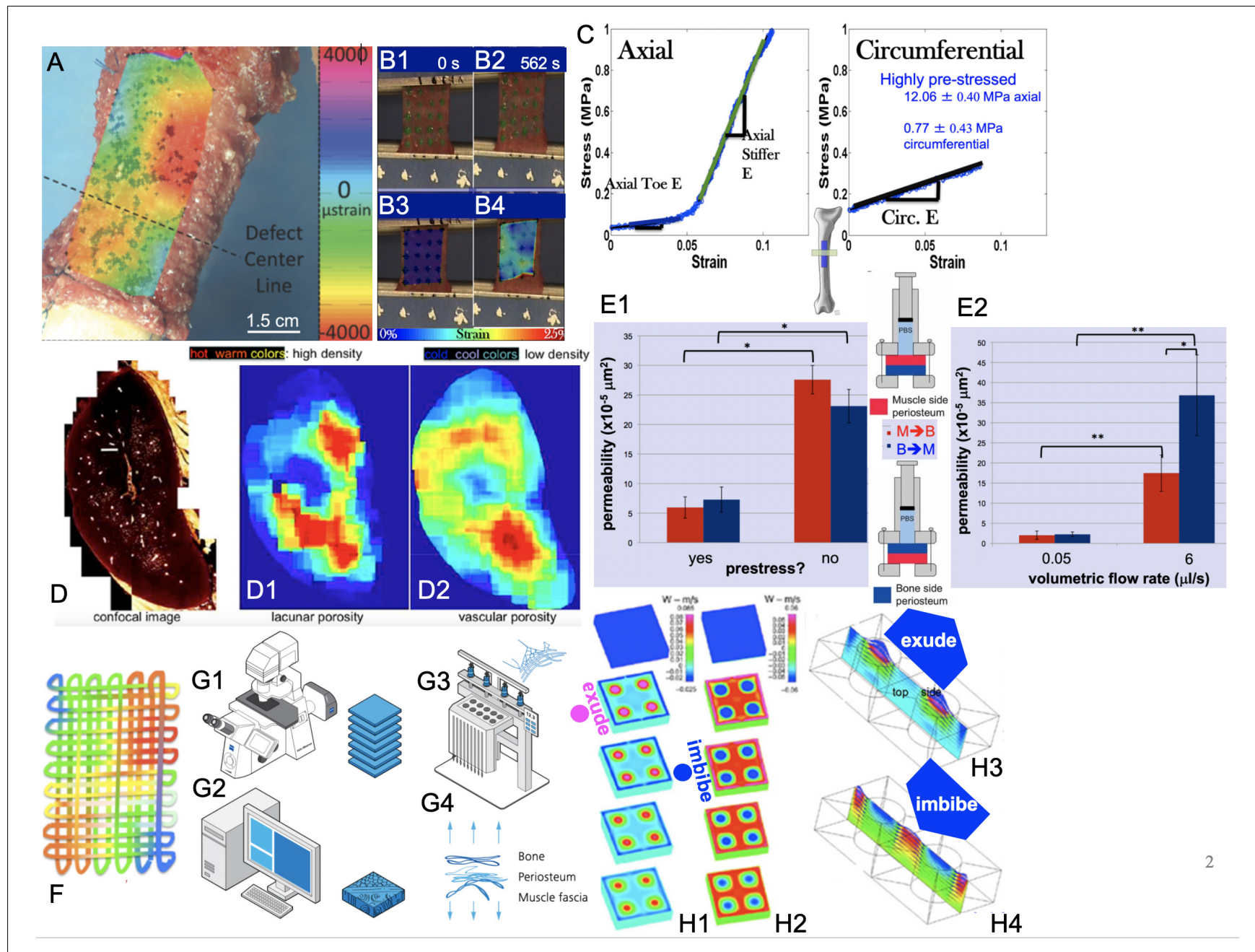

FIGURE 2 | Smart properties of mechanoactive materials, including skin-on-bones (periosteum, A,B,C,E) and bone itself (D) and MADAME approaches to emulate those properties (F,G1-G4,H1-H4). (A) Micron-resolution strain mapping of periosteum in situ under stance-shift loading demonstrates surprising heterogeneity (McBride et al., 2011a). (B1-B4) Similar heterogeneity is observed in strains during tensile testing of periosteum samples taken in the Axial and Circumferential directions (C) of the anterior (front) femur, which exhibit significant anisotropy (McBride et al., 2011b,c). (C - Axial) Specimens taken along the length of the femur exhibit strain stiffening while those taken along the circumference ( $\mathrm{C}$ - Circumferential) of the femur exhibit linear elasticity. Removal of the samples through cutting of the Sharpey's fibers and sample edges results in grossly observable shrinking of the specimens, which is significantly greater along the length of the long bone (Axial) compared to its circumference (McBride et al., 2011c). Based on shrinkage and measured moduli of elasticity, prestress of the tissue in situ can be calculated (Evans et al., 2013a). (D) Bone shows several calibers of porosity, including predominantly porosity around osteocytes (lacunar) and blood vessels (vascular). When the density of lacunar and vascular porosities are depicted spatially and as a heat map with warmer colors depicting higher density, the different patterns of lacunar (D1) and vascular (D2) densities are striking. Experimentally based computational models reveal counterintuitive flow effects when one explicitly accounts for these different spatial patterns of porosity densities, e.g., exudation of fluid under tension and imbibement of fluid under compression, which is contrary to daily experience with wet kitchen sponges (Reynolds, 1883; Knothe Tate et al., 2009). (E1,E2) Permeability studies on periosteum show a strong increase in permeability when prestress is removed from the tissue through cutting of Sharpey's fibers. In addition, permeability depends strongly on volumetric flow rate and direction of flow, where flow in the bone to muscle direction increases significantly more than that in the opposite direction with increase in volumetric flow rate; $p<0.05$ defines significant differences, * within, and ** between groups (Evans et al., 2013c). (F) Mechanical patterns resulting from intrinsic tissue weaves of e.g., elastin and collagen can be recreated recursively to create textiles emulating strain patterns observed in tissues. In this case, color patterns represent patterns of strain or fiber stiffness. Conceptually, "unraveling" of the tissue results in a singular solution with a fiber of varying stiffness along its length (Knothe Tate, 2016, 2017a,b, 2018; Ng et al., 2017c). An infinite number of solutions can be achieved through algorithms encoding patterns of stiffness, where microscopy acquired images (G1) provide a basis for infinite digital patterns and virtual prototypes (G2) that can be turned into physical prototypes using computer-controlled textile and knit systems, alone and/or in combination with other advanced manufacturing modalities such as multidimensional printing, laser sintering, etc. As a whole, the process is referred to as Microscopy-Aided Design And ManufacturE (Knothe Tate, 2016, 2018, 2019; Ng et al., 2017c). Figure adapted and compiled from previously published figures, with permission.

globular structures (mesenchyme, Figure 1B) that inter-convert throughout life. Indeed, all-natural tissue architectures are based on permutations of these sheets and globular structures, and development depends on the capacity of cells to revert between the two geometric design modalities, processes respectively referred to as epithelial-mesenchymal transition (EMT) and 
mesenchymal-epithelial transition (MET) (Morali et al., 2004; Knothe Tate et al., 2008, 2011a, 2016b; Evans et al., 2013c).

Tissues deriving developmentally from the epithelial sheets provide essential barrier functions to tissues at interfaces between different environments, e.g., skin provides a barrier between richly hydrated tissues of the body ( $~ 85 \%$ water by volume) and the outside environment and the skin-on-bones periosteum covers all non-collagenous bone surfaces of the body, separating the interior milieu from the outside environment of bone, e.g., muscle compartments, fat, etc. (Figures 1F, 2A,B). Tight junctions between cells of epithelial sheets underpin this barrier property with their interpenetrating, zipper-like closures between cell membranes (Knothe Tate, 2003; Morali et al., 2004; Knothe Tate et al., 2008; Evans et al., 2013c).

In contrast, more globular mesenchyme consists of cells and extracellular matrix, joined by a variety of cell-cell and cell matrix junctions that act as molecular rivets and more distributed Velcro-like attachments that each, respectively, attach to proteins of the cell's own skeleton (the cytoskeleton). Mesenchymal condensation, an event that occurs 11.5 days after fertilization in the mouse, initiates the formation of the musculoskeletal system. During this event, cells and their nascent tissues begin to specialize and rapidly scale-up cell number through cell division (proliferation) and formation of tissue templates through production of extracellular matrix (Morali et al., 2004; Knothe Tate et al., 2008; Evans et al., 2013c). Throughout the process, new geometries and architectures are stabilized by cell-cell and cell-matrix junctions.

Throughout prenatal development as well as during postnatal healing, which repeats prenatal development processes, cells interconvert between epithelial and mesenchymal states (EMTs and EMTs, as per above), enabling the formation of complex geometries, tissue architectures, and organ systems (Figures 1A,B; Morali et al., 2004; Putra et al., 2020). The process is so ubiquitous that the formation of deleterious tissues such as cancerous tumors and metastasis of circulating tumor cells follow analogous paradigms (Jolly et al., 2017). In many ways, this interconversion of geometries provides a most basic archetype for tissue architectures.

\section{Mechanical Modulation of Polarity to Anisotropy}

Just as mechanical forces modulate EMTs and METs as tissue architectures evolve, they also direct self assembly of cell layers at the earliest stages of tissue organization. Forces including cell adhesion and cell tension modulate tissue patterning of tissues and influence tissue phenotype from the time of fertilization until after birth and throughout life. As mechanisms by which forces inherent to life on Earth translate to self-assembly of multicellular structures, including tissues and organs, this knowledge provides direct inspiration for bottom-up design, engineering and manufacturing of mechanoactive materials and devices (Figures 1C, 2F,G; Knothe Tate et al., 2008). Also of great relevance, from the earliest stages of cell polarization to formation of materials exhibiting anisotropy, mechanical interactions and concentration gradients, at the interface of the cell and its environment, guide the transcription and secretion of structural proteins, the building blocks of tissues, in anisotropic structures that translate to anisotropic (in terms of mechanical properties) or directional functions and properties (Evans et al., 2013b).

\section{MECHANOACTIVE MATERIALS}

Electroactive polymers and materials change shape or size under electrical stimulation, while mechanoactive fibers and materials exhibit stimuli-responsive (smart) properties under mechanical loading (Figure 2). Given that life itself is mechanobiological, it is surprising that mechanoactive materials are less recognized and researched than electroactive materials. For example, if one compares search engine results for mechanoactive and electroactive materials, in PubMed, the former garner 14 compared to 4000 hits and, via google, 11,000 versus $2,200,000$ respective results. The plethora of electroactive materials and applications parallels industry and manufacturing activities around the development of electronics and electronic components, and their scale down in size over time. In the future, one would expect increased publication and patent activity around scaling down of advanced materials' and device manufacturing to include smart properties that emerge from smaller to larger length scales.

Interestingly, while top-down engineering approaches have led to elucidation of multiscale structure-function relationships in a variety of natural materials and tissue types (Spalazzi et al., 2008), bottom-up approaches appear more conducive to the elucidation, engineering and manufacture of emergent mechanoactive properties (Knothe Tate, 2011b; Evans et al., 2013c; Knothe Tate et al., 2016a). Emergence refers to properties or patterns that arise from the putting together of simpler elements which in themselves do not exhibit similar properties or patterns. The concept is further explained by example below.

\section{Periosteum, the Skin-on-Bones}

Periosteum, a hyper-elastic soft tissue sleeve, envelops all nonarticular (excluding the joint surfaces covered in cartilage) bony surfaces of the body, like a "skin-on-bones." As an inherently "smart" material, soft periosteum imparts hard bones with added failure strength under high impact loads (Evans et al., 2013a,b,c; Knothe Tate et al., 2016c). Recent studies of periosteum's mechanoactive properties reveal cellular and structural mechanisms underpinning its myriad smart properties, including anisotropic stiffness (modulus of elasticity) (McBride et al., 2011c), direction-dependent intrinsic prestress (McBride et al., 2011c; Yu et al., 2017) and permeability (Evans et al., 2013c), and a stem cell-triggering molecular weave that switches state upon release of prestress (Yu et al., 2017).

First, high definition television (HDTV) lenses were used to map in high resolution (submicron scale), four-dimensional (xyzt) strains in periosteum during stance shift loading of the sheep femur (McBride et al., 2011a,b). The rationale was to understand the local environment of stem cells that reside in a quiescent state in the periosteum until injury occurs. The working hypothesis was that the cells sense local strains 
in the periosteum which trigger them to egress from the periosteum in injury-inducing loading scenarios. Interestingly, $4 \mathrm{D}$-imaging of strains in the periosteum revealed surprising heterogeneity in space and time (Figure $\mathbf{2 A}$ and Supplementary Video 1). Furthermore, areas of new tissue genesis via stem cells egressing from the periosteum correlate to areas with the largest shift in baseline strain rather than absolute strain magnitudes (McBride et al., 2011a).

Further paired imaging-mechanics studies on sections of periosteum from the longitudinal (axial, along the long bone) and/or circumferential, anterior (front) aspect of the sheep femur demonstrated a stark anisotropy in mechanical properties of the periosteum (McBride et al., 2011c). When loaded in tension, axial oriented sections (Figures 2B,C-Axial), exhibit significant strain stiffening above 0.05 , while circumferentially oriented sections (Figures 2B,C-Circumferential) exhibit linear elastic behavior (McBride et al., 2011c).

Periosteum is attached to all bony surfaces via a plethora of collagen connections called Sharpey's fibers, which "Velcro" the soft tissue to the hard bony surface (Ng et al., 2017c; Yu et al., 2017). When injured, or during orthopedic surgeries involving periosteal lifting, the Sharpey's fibers become severed. In parallel with the mechanics studies described above, shrinkage of periosteum upon release from the underlying bone surface was quantified. Similar to mechanical stiffness, tissue shrinkage exhibited direction-dependence, whereby upon release, the tissue sections shrank significantly more in the axial compared to the circumferential direction. These relative shrinkages were used to calculate an intrinsic prestress in the tissue (Figure 2C), revealing that the tissue is highly prestressed in the axial direction $(12.06 \pm 0.40 \mathrm{MPa})$ and much less so in the circumferential direction $(0.77 \pm 0.43 \mathrm{MPa})$ (McBride et al., 2011a; Evans et al., 2013a).

Follow on high resolution microscope imaging studies revealed a potential mechanical trigger for quiescent stem cells to activate healing processes. By probing the interaction of light with the molecular structure of periosteum [second harmonic and two photon imaging (Ng et al., 2017c)], submicron resolution maps of collagen and elastin fibers in the tissue were rendered. When applied to freshly excised bone and periosteum in situ, it was observed that the intrinsic crimp or curl of collagen fibrils relaxes when the periosteum is released from the underlying bone via cutting of the Sharpey's fibers (Figure 1D). The relaxation in the curl was observed to coincide with rounding up of the resident stem cells adhering to the tissue fibers, providing a direct trigger for the cells to revert from quiescent to active states, and to initiate the genesis of new tissue templates associated with postnatal healing and prenatal development (Ng et al., 2017c).

Excitingly, the release of the tissue's intrinsic prestress had a significant impact on another property of the tissue, namely its permeability. Permeability is an essential functional boundary property (Figure 2E1) for periosteum, which serves as an interface between bone and surrounding muscle. Release of prestress in the tissue was associated with a significant reduction in permeability of periosteum. Furthermore, permeability of tissue-similar fluid (Ringer's lactate) through the periosteum exhibited direction- and flow-rate dependence. Permeability of periosteum increased eight to $16 \times$ when the flow rate was increased $120 \times$. Surprisingly, this effect was much more pronounced in the bone to muscle direction than in the muscleto-bone direction, a characteristic of a non-linear hydraulic valve (Evans et al., 2013a,b,c).

\section{Bone}

Further smart properties of bone reveal themselves when one analyzes the different phases of the tissue itself. During development in utero and during postnatal healing, bone starts as a soft template of collagen and elastin that mineralizes over time (Knothe Tate, 2011b; Ng et al., 2017c). The temporal aspect of bone mineralization and maturation can be tracked using fluorochromes of different excitation and emission wavelengths (Figure 1E; different spectra excite different fluorophores). The fluorochromes chelate chemically to the mineral when it is laid down onto (nucleates around the fibrils of) the bone template; intramuscular or subcutaneous injection of fluorochromes over the 4-month-healing cycle reveals the temporal dynamics of mineralization, e.g., of a tissue template formed via periosteum-derived stem cells ingressing into a bone defect (Figure 1E). Whereas initial mineralization forms a disorganized scaffold referred to as woven bone (red, Figure 1E), subsequent tissue genesis and mineralization occurs layer by layer, typically in proximity to the vascular supply (green and then turquoise, in time intervals comprising weeks, Figure 1E) (Knothe Tate et al., 2011b).

Intrigued by the observation of counterintuitive flows in experimentally based computational models of bones, we aimed to test the hypothesis that non-homogeneous distributions of different caliber pores in bone would result in such counterintuitive flows, e.g., imbibement of fluid under compression. Using high resolution microscopy, bone's different caliber porosities (pericellular versus vascular pores) were rendered as heat maps with warm colors depicting areas of high density and cool colors showing areas of low density (Figure 2D; Knothe Tate, 2003; Steck et al., 2003; Steck and Knothe Tate, 2005; Knothe Tate et al., 2009; Sidler et al., 2018). We then re-ran our computational models, which showed that specific patterns of porosity of different calibers indeed confer emergent, flowdirecting properties to the tissue under mechanical loads (Knothe Tate et al., 2011b).

\section{MICROSCOPY-AIDED DESIGN AND MANUFACTURE (MADAME) OF BIO-INSPIRED MATERIALS AND STRUCTURES}

\section{Concept - Recursive Logic to Emulate Natural Tissues}

Once we were able to quantify and precisely describe, quantitatively in four dimensions (4D), multiscale structure and emergent functional properties of natural materials, we then aimed to develop methods to design and manufacture new materials emulating nature's own. This resulted in the 
development of a novel process, referred to as MADAME, to map spatial and temporal properties of smart, natural materials (Figures 2F,G; Knothe Tate, 2017b; Sidler et al., 2018). The process uses imaging and advanced computational methods to visualize patterns intrinsic to the material (Figures 1, 2). Using recursive logic, the basis of computer algorithms, these patterns are recreated digitally using computer-aided design principles, and physically using computer-assisted weaving and/or knitting, alone and/or in combination with multidimensional additive manufacturing, e.g., 3D printing, stereolithography and laser sintering (Reynolds, 1883; McBride et al., 2008; Knothe Tate, 2016, 2017b; Yu et al., 2017).

The Jacquard loom was the earliest computer - in 1801, a century prior to "the first punch card driven computers, the Jacquard loom wove patterns using loops of paper with holes to guide when hooks fell through the paper loop (hook down) or stayed above the loop (hook up), thereby encoding binary patterns of e.g., tapestry weaves" (McBride et al., 2008; Song et al., 2013; Knothe Tate, 2016, 2017a; Sidler et al., 2018). The computer-controlled Jacquard looms and additive manufacturing systems enable creation of physical embodiments (textiles, composites) of mechanical and other biophysical and spatiotemporal patterns intrinsically encoded in natural materials.

More recent (2019) approaches (Sheiko and Dobrynin, 2019) suggest application of recursive logic for polymer design and engineering to mimic a range of mechanical properties appropriate for biological applications (Figure 3). While reduction to practice is currently in homogenous materials without intrinsic anisotropy or mechanical gradient properties (Vatankhah-Varnosfaderani et al., 2017), the concept expands upon the idea of encoding material properties architecturally, at molecular length scales, to achieve a range of biologically relevant mechanical properties previously achievable only empirically, through mixing of "various polymers, solvents and fillers" (Sheiko and Dobrynin, 2019). In combination with the aforementioned MADAME approach, a range of architectures might be achievable that range from molecular to micro- to macro- and mesolength scales. In particular, molecular encoding of polymers may provide a means to tune fiber and matrix mechanical properties for composite, advanced manufactured materials and products (Knothe Tate, 2019). In the future, it may enable real time manufacture of gradients materials through adjustment of the molecular composition of e.g., 3D printers and other advanced manufacturing platform materials.

\section{Incorporation of Pre- and Residual Stresses in Mechanoactive Material Design and Manufacture}

Once we implemented additive manufacturing with textile engineering to create composite structures, we aimed to integrate prestresses and/or residual stresses, found in natural materials, into advance-manufactured smart materials. A twopronged solution proved most effective. First we used a pre-tensioning system with the Jacquard loom to selectively prestress warp and weft (orthogonal weave) fiber directions
(Knothe Tate, 2017a; Knothe Tate and Thrasou, 2017). Then we incorporated principles of Kirigami, the Japanese art of folding and cutting paper into shapes, to cut and/or preform fibers and/or fiber composites with defined residual stresses and dimensions, from sheets or composites of desired materials (Knothe Tate, 2020). Stresses were thereby defined by the geometry of the cuts, e.g., spiral versus zig-zag with characteristic dimensions defining magnitudes of stresses and their gradients, or by the architecture of the composite fibers. While kirigami has been applied in electroactive materials (Cartolano et al., 2019), its use in mechanoactive materials is novel, in particular in combination with weaving, knitting and additive manufacturing applications. In developing these mechanisms and manufacturing strategies (below), the design thinking approach of empathizing with one's cells is particularly useful to brainstorm for new design elements and methods of reducing them to practice.

\section{Manufacturing Considerations}

Application of design thinking approaches that use the visualization tool of empathizing with cells that manufacture tissues, stimulates conceptualization of new manufacturing processes and/or pipelines. When one observes a range of natural architectures across phyla of the animal and plant kingdoms, from bone to corals (animal kingdom), and wide-ranging plants, the aforementioned geometric and/or directional/anisotropic paradigms are reiterated again and again, and thereby create an infinite number of structures and associated mechanoactive functions. In summary, empathizing with cells and understanding their unique structural and functional capacities facilitates development of new manufacturing methods and processes at different length and time scales (Knothe Tate et al., 2009, 2016a; Knothe Tate, 2011a, 2017b; Ng et al., 2017c, 2019). While surface based growth of biological structures is rapidly emulatable in additive manufacturing, new manufacturing modalities need to be developed further for other architectures and anisotropic properties. These manufacturing pipelines may incorporate steps typically used in composite structure and sandwich structure design, with modular assembly or they may involve novel incorporation of mechanoactive fiber assembly into textiles which themselves are assembled within photopolymerizable resin matrix and/or sintering powders. In other words, multimodal advanced manufacturing will likely follow in the footsteps of multimodal imaging, according to the MADAME paradigm described above. In materials not incorporating living cells, clever designs can be invented to actively modulate force balances at interfaces, much in the ways that cells do in living materials (Sidler et al., 2018).

\section{APPLICATIONS, FUTURE DIRECTIONS AND CONCLUSION}

Mechanoactive materials first found applications in tissue engineering for the development of tissue templates and scaffolds, as a direct translation from its inspiration (Anderson and Knothe Tate, 2007; Kuo and Tuan, 2008; Lujan et al., 2011; 

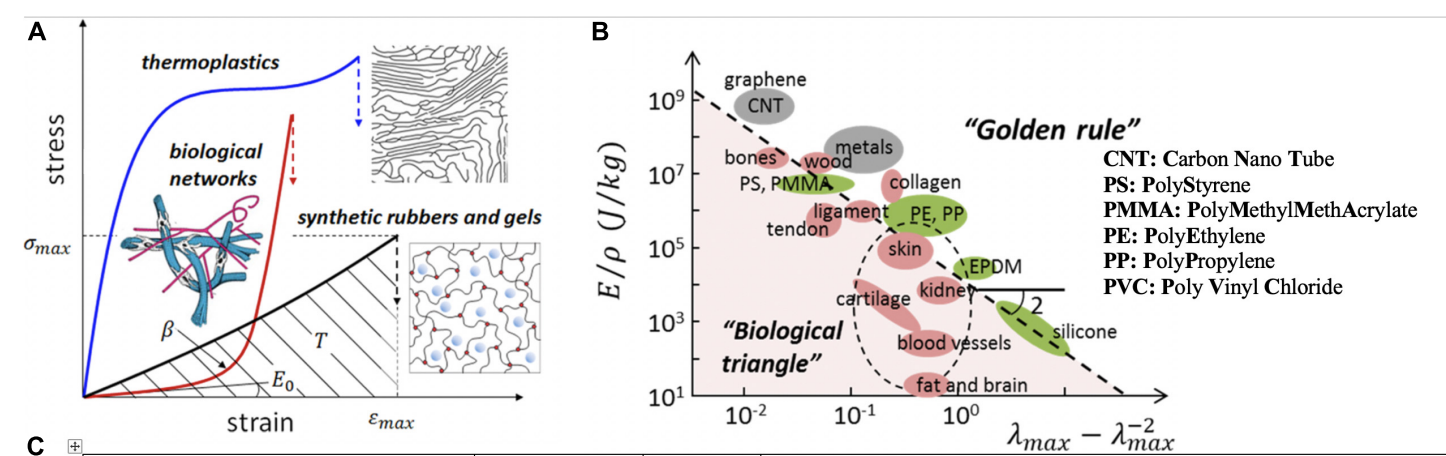

C

\begin{tabular}{|c|c|c|c|}
\hline $\begin{array}{c}\text { Material } \\
\text { Synthetic \& Biological materials } \\
\text { Textiles: warp - weft; grad is gradient }\end{array}$ & $\begin{array}{l}\text { Elastic Modulus } \\
\quad \pm S D(\mathrm{MPa})\end{array}$ & $\begin{array}{l}\text { Peak Force } \\
\text { (N) }\end{array}$ & Peak Strain - other notes \\
\hline $\begin{array}{c}\text { Compression sleeve textile } \\
72 \% \text { polyamide and } 28 \% \text { Lycra }{ }^{\mathbb{k}} \text { knit }\end{array}$ & $0.259-1.070$ & & Range of E, dependent on specimen alignment \\
\hline Skin & $0.425-1.05$ & & From [Agache et al., 1980] \\
\hline elastane - elastane & $0.85 \pm 0.04$ & $<10$ & $20 \%$ \\
\hline elastane - silk & $0.96 \pm 0.06$ & $<10$ & $20 \%$ \\
\hline Isotropic exercise bands & $0.88-1.5$ & & Range of $\mathrm{E}$ dependent on band grade (color) \\
\hline Transversalis fascia, human abdominal wall & $\sim 1.5$ & & Longitudinal samples; $\sim 0.7 \mathrm{MPa}$ ultimate tensile strength; from [Deeken and Lake, 2017] \\
\hline elastane - nylon & $1.73 \pm 0.29$ & $<10$ & $120 \%$ - hyperelastic \\
\hline Periosteum & 1.94 & & Low strain, $<5 \%$; longitudinal samples \\
\hline elastane - elastane & $2.26 \pm 0.15$ & $<10$ & $120 \%$ - hyperelastic, no hysteresis \\
\hline elastane - silk & $2.33 \pm 0.53$ & $<10$ & $120 \%$ - hyperelastic \\
\hline Transversalis fascia, human abdominal wall & $\sim 3$ & & Transverse samples; $\sim 1.5 \mathrm{MPa}$ ultimate tensile strength; from [Deeken and Lake, 2017] \\
\hline Periosteum & 4.41 & & Low strain, $<5 \%$; circumferential samples \\
\hline Periosteum & 25.67 & & Higher strain $>5 \%$; longitudinal samples \\
\hline Polybutester - Polybutester & $110 \pm 10$ & & \\
\hline grad $_{\text {silk }}$ grad $_{\text {clastane }}$ - elastane silk & $122 \pm 24$ & $>50$ & $9 \%$ \\
\hline ePTFE - ePTFE & $165 \pm 15$ & & \\
\hline nylon - nylon & $200.44 \pm 7.52$ & $>250$ & $20 \%$ - slippage of sample at Peak Force \\
\hline silk - silk grad elastane & $280 \pm 22$ & $>150$ & $9 \%$ \\
\hline nylon - elastane & $328.55 \pm 31.28$ & $>250$ & $20 \%$ - slippage of sample at Peak Force \\
\hline silk - elastane & $370 \pm 78$ & $>250$ & \\
\hline silk - silk & $443.19 \pm 94.86$ & $>400$ & $22 \%-$ load cell limit reached \\
\hline silk - elastane & $480.24 \pm 70.76$ & $>400$ & $22 \%$ - load cell limit reached \\
\hline Flexible $P V C$ used in i.v. bags, medical tubing & circa $1-1800$ & & $\begin{array}{l}\text { SpecialChem, Comprehensive Guide onPolyvinyl Chloride (PVC), Omnexus, } 2017 . \\
\text { https://omnexus.specialchem.com/selectionguide/polyvinyl-chloride-pvc-plastic }\end{array}$ \\
\hline
\end{tabular}

FIGURE 3 | Sheiko and Dobrynin's concept of encoding mechanical phenotype in synthetic polymeric materials and Knothe Tate's recursive logic approach to encoding mechanoactive material properties in textile (and composite) patterns comprising fibers of different mechanical phenotypes. (A) Thermoplastics, synthetic elastomers and gels and biological networks (extracellular matrix, tissue) exhibit mechanical phenotypes exemplified through the materials' stress $\sigma$ - strain $\varepsilon$ curve $\left(E_{0}\right.$, the Young's Modulus, is the material stiffness in the linear elastic region of the curve; $\beta$ shows strain stiffening or non-linear increase in Modulus with increase in deformation, a measure of material firmness; $\sigma_{\max }$ is the strength of the material or stress at break; the area under the curve indicates material toughness or how much energy is dissipated at breakage. (B) Elongation at break $\left(\lambda_{\max }\right)$ versus Young's modulus $(E)$ over the mass density $(\rho)$ of a material. "This representation recovers scaling relations $\mathrm{E} \sim \lambda_{\max }-2$, for elastomeric materials with $\lambda_{\max }{ }^{\prime 1}$, and $\mathrm{E} \sim \varepsilon_{\max }-2$, for hard materials with strain at break $\varepsilon_{\max }=\lambda_{\max }-1$ «1." Panels (A,B) used with permission from Sheiko and Dobrynin (2019) and Vatankhah-Varnosfaderani et al. (2017). (C) Master Table of properties for materials created using MADAME and tested in tension, compared to textile and isotropic control materials, using data from Ng et al. (2017a,b,c, 2019, 2020).

Song et al., 2013; Cartolano et al., 2019). In parallel, the mechanoactive materials were introduced for internal (implants) and external medical devices, mainly in cardiovascular and orthopedic fields of use (Knothe Tate et al., 2011a; Ng et al., 2020). New classes of mechanoactive materials will find uses i.a. in the medical and health sectors ( $\mathrm{Ng}$ et al., 2017a; Hageman et al., 2018), in addition to safety and transport, military (Knothe Tate, 2011a), sports, and leisure wear sectors, cementing their disruptive status in the field of material science and advanced manufacturing (Knothe Tate et al., 2009; Sidler et al., 2018).

While biomimicry has inspired artists, scientists and engineers for well over five centuries ( $c f$. da Vinci's flying machine), the advent of advanced imaging has provided a critical enabling technology to decipher mechanisms underpinning emergent properties of natural materials and systems at multi-length and -time scales (Knothe Tate, 2017a,b). Such advances enabled through imaging have forged a path for translation of knowledge via design and manufacture of materials emulating the emergent properties of nature's own (Knothe Tate, 2017b; Sidler et al., 2018). Such properties integrate sensor, transducer and actuator functions in the material itself (Knothe Tate et al., 2016b; Sidler et al., 2018).

Microscopy-Aided Design And ManufacturE lends itself well for the deciphering, design and manufacture of emergent properties from nature (Sidler et al., 2018; Ng et al., 2019, 2020). This is expected to result in parallel advances in innovative technological solutions and manufacturing of new materials and products harnessing those solutions. Visualization through high resolution spatiotemporal imaging is particularly enabling for mechanoactive materials, as the field of mechanics has a rich history of translating visualization of mechanical properties to elucidation of mechanisms and new inventions.

Similitude theory implements parametric scaling to study mechanics in very large- or small-scale systems where direct measures were not possible with then state-of-the-art technology (da Vinci, 1508; Einstein, 1911; Bridgman, 1922; Astarita, 1997; Anderson et al., 2008). For example, in aeronautics, an airplane 
or wing was typically scaled down in size to a model system on which flow parameters could be measured experimentally which would be impossible on the true-to-scale system. The scaling relationship enables linking of the model parameters to those of the actual system. In fluid dynamics, "qualitative and quantitative descriptions of flow regimes can be obtained using scaling relationships if viscous and inertial forces (Reynolds number) are maintained across the length scales (Reynolds, 1883, 1895; Rayleigh, 1892, 1904, 1915; Anderson et al., 2008; Sidler et al., 2018; Sheiko and Dobrynin, 2019).

In combination with MADAME, similitude theory may lead to new fundamental discoveries as well as design approaches and manufacturing methods in previously intractable nanomicro biological systems, enabling visualization of biophysical phenomena below the traditional realm of visualization (Anderson et al., 2008). In this way, MADAME serves as an enabling technology that, in combination with centuryold theories such as similitude, may open the path toward new discoveries and inventions while also providing a path to manufacture materials and products with bio-inspired properties and mechanisms.

\section{DISCLOSURE}

MK has intellectual property patented and pending patent around multilayered surgical membranes as well as the design and manufacture of advanced manufactured composites that emulate nature's own. The commercialization of these technologies is at an early (pre-revenues generating) stage. This manuscript reports scientific outcomes designed to benefit the field as a whole and does not report on any particular product or prototype with potential commercial interest.

\section{REFERENCES}

Agache, P. G., Monneur, C., Leveque, J. L., and De Rigal, J. (1980). Mechanical properties and young's modulus of human skin in vivo. Archiv. Dermatol. Res. 269, 221-232. doi: 10.1007/BF00406415

Anderson, E. J., and Knothe Tate, M. L. (2007). Design of tissue engineering scaffolds as delivery devices for mechanical and mechanically modulated signals. Tissue Eng. 13, 2525-2538. doi: 10.1089/ten.2006.0443

Anderson, E. J., Kreuzer, S. M., Small, O., and Knothe Tate, M. L. (2008). Pairing computational and scaled physical models to determine permeability as a measure of cellular communication in micro- and nano-scale pericellular spaces. Microfluid. Nanofluidics 4, 193-204. doi: 10.1007/s10404-007-0156-5

Astarita, G. (1997). Dimensional analysis, scaling, and orders of magnitude. Chem. Eng. Sci. 52, 4681-4698. doi: 10.1016/s0009-2509(97)85420-6

Bridgman, P. (1922). Dimensional Analysis. New Haven: Yale University Press.

Cartolano, M., Xia, B., Miriyev, A., and Lipson, H. (2019). Conductive fabric heaters for heat-activated soft actuators. Actuators 8:9. doi: 10.3390/act8010009

da Vinci, L. (1508). in Pilot Plants, Models, and Scale up Methods in Chemical Engineering, eds R. E. Johnstone and M. W. Thring (New York, NY: McGrawHill).

Deeken, C. R., and Lake, S. P. (2017). Mechanical properties of the abdominal wall and biomaterials used for hernia repair. J. Mech. Behav. Biomed. Mater. 74, 411-427. doi: 10.1016/j.jmbbm.2017.05.008

Einstein, A. (1911). Elementare Betrachtungen über die thermische Molekularbewegung in festen Körpern. Ann. Phys. 35, 679-694. doi: 10.1002/andp.19113400903

\section{ETHICS STATEMENT}

The animal study was reviewed and approved by the Commission for the Ethical Use and Care of Animals, Grisons, Switzerland.

\section{AUTHOR CONTRIBUTIONS}

MK wrote the perspective article which reflects her perspectives on the field.

\section{FUNDING}

Parts of this study were funded by the National Institutes of Health, National Science Foundation, Wallace H. Coulter Foundation, AO Foundation, Christopher Columbus United States Commerce Department, National Health and Medical Research Council, and the Paul Trainor Foundation.

\section{ACKNOWLEDGMENTS}

Supplementary Video 1 was created by Sarah Gagyi-McBride as part of a previous study (McBride et al., 2011a). It has not been published in video format previously.

\section{SUPPLEMENTARY MATERIAL}

The Supplementary Material for this article can be found online at: https://www.frontiersin.org/articles/10.3389/fbioe. 2020.00845/full\#supplementary-material

Evans, S. F., Chang, H., and Knothe Tate, M. L. (2013a). Elucidating multiscale periosteal mechanobiology: a key to unlocking the regenerative capacity of the periosteum? Tissue Eng. Part B Rev. 19, 147-159. doi: 10.1089/ten.teb.2012.0216

Evans, S. F., Docheva, D., Bernecker, A., Colnot, C., Richter, R. P., and Knothe Tate, M. L. (2013b). Solid-supported lipid bilayers to drive stem cell fate and tissue architecture using periosteum derived progenitor cells. Biomaterials 34, 1878-1887. doi: 10.1016/j.biomaterials.2012.09.024

Evans, S. F., Parent, J. B., Lasko, C. E., Zhen, X., Knothe, U. R., Lemaire, T., et al. (2013c). Periosteum, Bone's "Smart" bounding membrane, exhibits direction dependent permeability. J. Bone Miner. Res. 28, 608-617. doi: 10.1002/jbmr. 1777

Hageman, D. J., Wu, S., Kilbreath, S., Rockson, S. G., Wang, C., and Knothe Tate, M. L. (2018). Biotechnologies toward mitigating, curing, and ultimately preventing edema through compression therapy. Trends Biotechnol. 36, 537548. doi: 10.1016/j.tibtech.2018.02.013

Jolly, M. K., Ware, K. E., Gilja, S., Somarelli, J. A., and Levine, H. (2017). EMT and MET: necessary or permissive for metastasis? Mol. Oncol. 11, 755-769. doi: 10.1002/1878-0261.12083

Knothe Tate, M. L. (2003). "Whither flows the fluid in bone?": an Osteocyte's Perspective. J. Biomech. 36, 1409-1424. doi: 10.1016/S0021-9290(030)00123-4

Knothe Tate, M. L. (2011a). Smart body armor inspired by flow in bone. Smart Struct. Syst. 7, 223-228. doi: 10.12989/sss.2011.7.3.223

Knothe Tate, M. L. (2011b). Top down and bottom up engineering of bone. J. Biomech. 44, 304-312. doi: 10.1016/j.jbiomech.2010.10.019

Knothe Tate, M. L. (2016). Engineered Materials and Methods of Forming. U.S. Patent Application based on United States Provisional Patent Application Serial 
No. 62/175,842. PCT Application No. PCT/IB2016/053553. Washington, DC: U.S. Patent and Trademark Office.

Knothe Tate, M. L. (2017a). Biotextilogy - The Cellular Catwalk. Coogee: Melissa L. Knothe Tate.

Knothe Tate, M. L. (2017b). "Navigation of bee brains to human hips - microscopy and the modern Magellans," in A New Age in Scanning Electron Microscopy: Applications in the Life Sciences (Washington, DC: Science/ AAAS), 19-23.

Knothe Tate, M. L. (2018). Prospective Design of Combination Products/Delivery Devices Exploiting Multiphysical Mechanisms for Wound Healing. US Patent Application Serial No. 62/501,537. Washington, DC: U.S. Patent and Trademark Office.

Knothe Tate, M. L. (2019). Smart Composite Textiles and Methods of Forming. U.S. PCT/IB2019/053708. Washington, DC: U.S. Patent and Trademark Office.

Knothe Tate, M. L. (2020). Design and Manufacture of Mechanoactive Materials. U.S. Provisional Patent 62/984,660. Washington, DC: U.S. Patent and Trademark Office.

Knothe Tate, M. L., and Anderson, E. J. (2013). Flow Directing Materials and Systems. U.S. Patent 12/106.748. Washington, DC: U.S. Patent and Trademark Office.

Knothe Tate, M. L., Chang, H., Moore, S. R., and Knothe, U. R. (2011a). Surgical membranes as directional delivery devices to generate tissue in critical sized defects. PLoS One 6:e28702. doi: 10.1371/journal.pone.0028702

Knothe Tate, M. L., Dolejs, S., McBride, S. H., Miller, R. M., and Knothe, U. R. (2011b). Multiscale Mechanobiology of De Novo bone generation as well as remodeling \& adaptation of autograft - an integrative review. J. Mech. Behav. Biomed. Mater. 4, 829-840. doi: 10.1016/j.jmbbm.2011.03.009

Knothe Tate, M. L., Detamore, M., Capadona, J. R., Wooley, A., and Knothe, U. (2016a). Engineering and commercialization of human-device interfaces. from bone to brain. Biomaterials 95, 35-46. doi: 10.1016/j.biomaterials.2016. 03.038

Knothe Tate, M. L., Gunning, P. W., and Sansalone, V. (2016b). Emergence of form from function: role of stem cell mechanoadaptation in sealing of cell fate. BioArchitecture 6, 85-103. doi: 10.1080/19490992.2016. 1229729

Knothe Tate, M. L., Yu, N. C., Jalilian, I., Pereira, A. F., and Knothe, U. R. (2016c). Periosteum mechanobiology and mechanistic insights for regenerative medicine. Nat. Publ. Group Bonekey Rep. 5:857. doi: 10.1038/bonekey.2016.70

Knothe Tate, M. L., Falls, T., Mishra, S., and Atit, R. (2010). "Engineering an ecosystem: taking cues from nature's paradigm to build tissue in the lab and the body," in New Perspectives in Mathematical Biology, ed. S. Sivaloganathan (Providence, RI: American Mathematical Society), 113-133.

Knothe Tate, M. L., Falls, T. D., McBride, S. H., Atit, R., and Knothe, U. R. (2008). Mechanical modulation of osteochondroprogenitor cell fate. Int. J. Biochem.Cell Biol. 40, 2720-2738. doi: 10.1016/j.biocel.2008.05.011

Knothe Tate, M. L., and Fath, T. (2016). The only constant is change: next generation medical devices for physical and mental health. Adv. Healthc. Mater. 5, 1840-1843. doi: 10.1002/adhm.201600437

Knothe Tate, M. L., and Niederer, P. (1998). "A theoretical FE-based model developed to predict the relative contribution of convective and diffusive transport mechanisms for the maintenance of local equilibria within cortical bone. HTD," in Advances in Heat and Mass Transfer in Biotechnology, Vol. 362/BED - Vol. 40. ed. S. Clegg (New York, NY: The American Society of Mechanical Engineers), 133-142.

Knothe Tate, M. L., Steck, R., and Anderson, E. J. (2009). Bone as an inspiration for a novel class of biomaterials. Biomaterials 30, 133-140. doi: 10.1016/j. biomaterials.2008.09.028

Knothe Tate, M. L., and Thrasou, P. (2017). Loom Fiber Tensioning Device. U.S. Provisional Patent 62/577,039. Washington, DC: U.S. Patent and Trademark Office.

Kuo, C. K., and Tuan, R. S. (2008). Mechanoactive tenogenic differentiation of human mesenchymal stem cells. Tissue Eng. Part A 14, 1615-1627. doi: 10.1089/ ten.tea.2006.0415

Lopez, R., Badel, E., Peraudeau, S., Leblanc-Fournier, N., Beaujard, F., Julien, J.L., et al. (2014). Tree shoot bending generates hydraulic pressure pulses: a new long-distance signal? J. Exp. Bot. 65:1997. doi: 10.1093/jxb/eru045

Lujan, T. J., Wirtz, K. M., Bahney, C. S., Madey, S. M., Johnstone, B., and Bottlang, M. (2011). A novel bioreactor for the dynamic stimulation and mechanical evaluation of multiple tissue-engineered constructs. Tissue Eng. Part C Methods 17, 367-374. doi: 10.1089/ten.TEC.2010.0381

McBride, S. H., Dolejs, S., Brianza, S., Knothe, U., and Knothe Tate, M. L. (2011a). Net change in periosteal strain correlates to rapid De Novo bone generation in critical sized defects. Ann. Biomed. Eng. 39, 1570-1581. doi: 10.1007/s10439010-0242-9

McBride, S. H., Dolejs, S., Knothe, U., and Knothe Tate, M. L. (2011b). Major and minor centroidal axes serve as objective, automatable reference points to test mechanobiological hypotheses using histomorphometry. J. Biomech. 44, 1205-1208. doi: 10.1016/j.jbiomech.2011.01.033

McBride, S. H., Evans, S. F., and Knothe Tate, M. L. (2011c). Anisotropic mechanical properties of ovine femoral periosteum and the effects of cryopreservation. J. Biomech. 44, 1954-1959. doi: 10.1016/j.jbiomech.2011. 04.036

McBride, S. H., Falls, T., and Knothe Tate, M. L. (2008). Modulation of stem cell shape and fate, B: mechanical modulation of cell shape and gene expression. Tissue Eng. 14, 1573-1580. doi: 10.1089/ten.tea.2008.0113

Mishra, S., and Knothe Tate, M. L. (2003). Effect of lacunocanalicular architecture on hydraulic conductance in bone tissue: implications for bone health and evolution. Anat. Rec. 273A, 752-762. doi: 10.1002/ar.a.10079

Moore, S. R., Heu, C., Yu, N. Y. C., Whan, R., Knothe, U. R., Milz, S., et al. (2016). Translating Periosteum"s regenerative power: insights from quantitative analysis of tissue genesis with a periosteum substitute implant. Stem Cells Transl. Med. 5, 1739-1749. doi: 10.5966/sctm.2016-0004

Moore, S. R., Milz, S., and Knothe Tate, M. L. (2014). The linea aspera: a virtual case study testing emergence of form and function. Anat. Rec. 297, 273-280. doi: 10.1002/ar.22840

Morali, O., Savagner, P., and Larue, L. (2004). "Epithelium - mesenchyme transitions are crucial morphogenetic events occurring during early development. Chapter 2," in Rise and Fall of the Epithelial Phenotype, ed. P. Savagner (Amsterdam: Kluwer Academic/Plenum Publishers).

Ng, J. L., Collins, C. E., and Knothe Tate, M. L. (2017a). Engineering mechanical gradients in next generation biomaterials - lessons learned from medical textile design. Acta Biomater. 56, 14-24. doi: 10.1016/j.actbio.2017. 03.004

Ng, J. L., Kersh, M. E., Kilbreath, S., and Knothe Tate, M. L. (2017b). Establishing the basis for mechanobiology-based physical therapy protocols to potentiate cellular healing and tissue regeneration. Front. Physiol. Exerc. Physiol. 8:303. doi: $10.3389 /$ fphys.2017.00303

Ng, J. L., Knothe, L., Whan, R., Knothe, U., and Knothe Tate, M. L. (2017c). Scale-up of nature's tissue weaving algorithms to engineer advanced functional materials. Sci. Rep. 7:40396. doi: 10.1038/srep40396

Ng, J. L., Putra, V. D. L., and Knothe Tate, M. L. (2020). Biocompatibility and biomechanics studies of woven textiles to emulate natural tissue weaves and their intrinsic gradients. J. Mech. Behav. Biomed. Mat. 103:103536. doi: 10.1016/ j.jmbbm.2019.103536

Ng, J. L., Singh, T., and Kwan, L. C. (2019). Biotextilogy - Prototyping and testing mechanical gradient textiles that emulate nature's own. Results Mater. 2:100018. doi: 10.1016/j.rinma.2019.100018

Putra, V. D. L., Song, M. J., McBride-Gagyi, S., Chang, H., Poole, K., Whan, R., et al. (2020). Mechanomics approaches to understand cell behavior in context of tissue neogenesis, during prenatal development and postnatal healing. Front. Cell. Dev. Biol. 7:354. doi: 10.3389/fcell.2019.0 0354

Rayleigh, L. (1892). On the question of the stability of the flow of fluids. Philos. Mag. 34, 59-70.

Rayleigh, L. (1904). Fluid friction on even surfaces. Philos. Mag. 8, 66-67.

Rayleigh, L. (1915). The principle of similitude. Nature 95, 66-68.

Reynolds, O. (1883). An experimental investigation of the circumstances which determine whether the motion of water shall be direct or sinuous, and of the law of resistance in parallel channels. Philos. Trans. R. Soc. Lond. 174, 935-982. doi: 10.1098/rstl.1883.0029

Reynolds, O. (1895). On the dynamical theory of incompressible viscous fluids and the determination of the criterion. Philos. Trans. R. Soc. Lond. 186, 123-164. doi: 10.1098/rsta.1895.0004

Sheiko, S. S., and Dobrynin, A. V. (2019). Architectural code for rubber elasticity, from supersoft to superfirm materials. Macromolecules 52, 7531-7546. doi: 10.1021/acs.macromol.9b01127 
Sidler, H. J., Duvenage, J., Anderson, E. J., Ng, J., Hageman, D. J., and Knothe Tate, M. L. (2018). Prospective design, rapid prototyping and testing of smart dressings, drug delivery patches, and replacement body parts using microscopy aided design and manufacturE (MADAME). Front. Med. 5:348. doi: 10.3389/ fmed.2018.00348

Song, M. J., Dean, D., and Knothe Tate, M. L. (2013). Mechanical modulation of nascent cell lineage commitment in tissue engineering scaffolds. Biomaterials 34, 5766-5775. doi: 10.1016/j.biomaterials.2013.04.023

Spalazzi, J. P., Vyner, M. C., Jacobs, M. T., Moffat, K. L., and Lu, H. H. (2008). Mechanoactive scaffold induces tendon remodelling and expression of fibrocartilage markers. Clin. Orthop. Relat. Res. 466, 1938-1948. doi: 10.1007/ s11999-008-0310-8

Steck, R., and Knothe Tate, M. L. (2005). In silico stochastic network models that emulate the molecular sieving characteristics of bone. Ann. Biomed. Eng. 33, 87-94. doi: 10.1007/s10439-005-8966-7

Steck, R., Niederer, P., and Knothe Tate, M. L. (2000). A Finite difference model of load-induced fluid displacements within bone under mechanical loading. Med. Eng. Phys. 22, 117-125. doi: 10.1016/S1350-4533(00)00 017-5

Steck, R., Niederer, P., and Knothe Tate, M. L. (2003). A finite element analysis for the prediction of load-induced fluid flow and mechanochemical transduction in bone. J. Theor. Biol. 220, 249-259. doi: 10.1006/jtbi.2003.3163

Tee, B. C.-K., Wang, C., Allen, R., and Bao, Z. (2012). An electrically and mechanically self-healing composite with pressure- and flexion-sensitive properties for electronic skin applications. Nat. Nanotech 7, 825-832. doi: 10 . 1038/nnano.2012.192

Vatankhah-Varnosfaderani, M., Daniel, W. F. M., Everhart, M. H., Pandya, A. A., Liang, H., Matyjaszewski, K., et al. (2017). Mimicking biological stressstrain behaviour with synthetic elastomers. Nature 549, 497-501. doi: 10.1038/ nature23673

Yu, N. Y. C., O’Brien, C. A., Slapetova, I., Whan, R. M., and Knothe Tate, M. L. (2017). Live tissue imaging to elucidate mechanical modulation of stem cell niche quiescence. Stem Cells Transl. Med. 6, 282-292. doi: 10.5966/sctm.20150306

Conflict of Interest: MK is the inventor of several patented and patent pending technologies (Knothe Tate, 2016, 2018, 2019) and has an interest in a start-up company that was formed to commercialize these technologies. The pre-revenues stage start-up company is focused on $\mathrm{R} \& \mathrm{D}$, and the current manuscript reflects the scientific findings of this R\&D work in addition to the MK's university-based laboratory endeavors.

Copyright (c) 2020 Knothe Tate. This is an open-access article distributed under the terms of the Creative Commons Attribution License (CC BY). The use, distribution or reproduction in other forums is permitted, provided the original author(s) and the copyright owner(s) are credited and that the original publication in this journal is cited, in accordance with accepted academic practice. No use, distribution or reproduction is permitted which does not comply with these terms. 